PDES, SUBMANIFOLDS AND

AFFINE DIFFERENTIAL GEOMETRY

BANACH CENTER PUBLICATIONS, VOLUME 69

INSTITUTE OF MATHEMATICS

POLISH ACADEMY OF SCIENCES

WARSZAWA 2005

\title{
FAMILIES OF CONGRUENT SUBMANIFOLDS
}

\author{
SEBASTIAN KLEIN and HELMUT RECKZIEGEL \\ Mathematisches Institut der Universität zu Köln \\ Weyertal 86-90, 50931 Köln, Germany \\ E-mail:sklein@math.uni-koeln.de,reckziegel@math.uni-koeln.de
}

Among the families of congruent totally geodesic submanifolds that occur in an $\mathrm{m}$ dimensional projective complex quadric $Q$, there are two types which are of particular interest: the family of $k$-dimensional projective subspaces $(k \leq m / 2)$ contained in $Q$ (that such subspaces exist is well-known from algebraic geometry, see for example [GH], Proposition 6.1 , p. 735), and the family of $k$-dimensional complex quadrics $(k<m)$ which are totally geodesic in $Q$. The primary subject of this article is to give these families the structure of a Riemannian manifold and to study them, in particular as submanifolds of the families of all $k$-dimensional projective subspaces resp. complex quadrics contained in the ambient projective space $\mathbb{P}^{m+1}$. A large part of this study is focused on questions from the theory of reductive homogeneous spaces and of symmetric spaces. In particular, we show in a general setting that a family of congruent manifolds can be seen as a naturally reductive homogeneous space. In the specific cases mentioned above, we investigate whether the reductive structure of the families is induced by a symmetric structure, and whether the families in $Q$ are naturally reductive homogeneous subspaces of the corresponding families in $\mathbb{P}^{m+1}$.

In Section 1, we present fundamental facts on families of congruent homogeneous subspaces in Riemannian homogeneous spaces in general. Section 2 is concerned with results on congruence families of projective subspaces and of quadrics in a projective space, and Section 3 finally discusses the corresponding families of projective subspaces and of quadrics which are contained in a fixed quadric $Q$.

2000 Mathematics Subject Classification: Primary 53C35; Secondary 53C17.

The second author would like to thank K. Kenmotsu for his invitation to the Tôhoku University (Sendai) in May 2002 and his hospitality. There he met C. Gherghe (Bucharest), from whom he learned the relation between Theorem 8 and twistor spaces, for instance see [Ra].

The paper is in final form and no version of it will be published elsewhere. 


\section{Families of congruent submanifolds}

1.1. Families of submanifolds and their differentiable structure. Let $M$ be a Riemannian homogeneous space, $G:=I(M)$ the isometry group of $M$, and $N_{0}$ a connected, closed homogeneous subspace of $M$, i.e. the group $K:=\left\{f \in G \mid f\left(N_{0}\right)=N_{0}\right\}$ acts transitively on $N_{0}$. In this situation, $K$ is closed in $G$, and therefore a Lie subgroup of $G$, and $N_{0}$ is a regular ${ }^{1}$ submanifold of $M$.

Then we call the set $\mathfrak{F}\left(N_{0}, M\right):=\left\{f\left(N_{0}\right) \mid f \in G\right\}$ of submanifolds of $M$ the congruence family of submanifolds induced by $N_{0}$. For a fixed $p \in M$, we also consider the subfamily $\mathfrak{F}_{p}\left(N_{0}, M\right):=\left\{N \in \mathfrak{F}\left(N_{0}, M\right) \mid p \in N\right\}$.

Proposition 1. (a) There is a unique differentiable structure on $\mathfrak{F}\left(N_{0}, M\right)$, such that the transitive action $\varphi: G \times \mathfrak{F}\left(N_{0}, M\right) \rightarrow \mathfrak{F}\left(N_{0}, M\right),(f, N) \mapsto f(N)$ is differentiable.

(b) For every $p \in M, \mathfrak{F}_{p}\left(N_{0}, M\right)$ is a compact, regular submanifold and a homogeneous subspace of $\mathfrak{F}\left(N_{0}, M\right)$ with respect to the action of the isotropy group $G_{p}$ of $M$ at $p$ on $\mathfrak{F}_{p}\left(N_{0}, M\right)$.

(c) For every $p \in M$ and $f \in G$, the map $\Sigma: G_{p} \rightarrow G_{f(p)}, g \mapsto f \circ g \circ f^{-1}$ is an isomorphism of Lie groups. The map $\sigma: \mathfrak{F}_{p}\left(N_{0}, M\right) \rightarrow \mathfrak{F}_{f(p)}\left(N_{0}, M\right), N \mapsto f(N)$ is a diffeomorphism and the pair $(\sigma, \Sigma)$ is an isomorphism of homogeneous spaces, meaning that the diagram

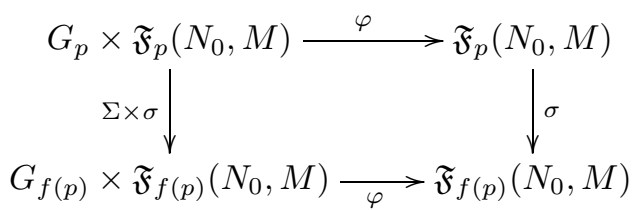

commutes. $^{2}$

Proof. Because $K$ is closed in $G$, the quotient $G / K$ carries the structure of a differentiable manifold ([V], Theorem 2.9.4, p. 77), which we transfer onto $\mathfrak{F}\left(N_{0}, M\right)$ by the $G$-equivariant bijection $G / K \rightarrow \mathfrak{F}\left(N_{0}, M\right), f \cdot K \mapsto f\left(N_{0}\right)$. The uniqueness statement is also proved in $[\mathrm{V}]$, Theorem 2.9.4.

Because $N_{0}$ is a homogeneous subspace of $M, G_{p}$ acts transitively on $\mathfrak{F}_{p}\left(N_{0}, M\right)$ and is compact (see $[\mathrm{K}]$, Theorem II.1.2, p. 39). Therefore, $\mathfrak{F}_{p}\left(N_{0}, M\right)$ is a regular submanifold and a homogeneous subspace of $\mathfrak{F}\left(N_{0}, M\right)$. Statement (c) is obvious.

1.2. The relation between the dimensions of $\mathfrak{F}_{p}\left(N_{0}, M\right)$ and $\mathfrak{F}\left(N_{0}, M\right)$

Proposition 2. In the situation of the previous section we fix some $p_{0} \in N_{0}$.

\footnotetext{
${ }^{1}$ We call a submanifold $N$ of a manifold $M$ regular if the intrinsic topology of $M$ coincides with the topology inherited from $M$; see [V], p. 18.

${ }^{2}$ If, in this setting, $\Sigma$ only is a homomorphism of Lie groups and $\sigma$ a differentiable map, we call the pair $(\sigma, \Sigma)$ a homomorphism of homogeneous spaces. If $(\sigma, \mathrm{id})$ is a homomorphism of homogeneous spaces, we also call $\sigma$ simply a homomorphism of homogeneous spaces. If the homogeneous spaces involved are equipped with reductive structures, and $\Sigma$ respects these structures, we call $(\sigma, \Sigma)$ a homomorphism (isomorphism) of reductive homogeneous spaces.
} 
(a) $\operatorname{dim} \mathfrak{F}_{p_{0}}\left(N_{0}, M\right)=\operatorname{dim} G_{p_{0}}-\operatorname{dim}\left(G_{p_{0}} \cap K\right)$.

(b) If $N_{0}$ is a complete, totally geodesic submanifold of $M$, we have $G_{p_{0}} \cap K=\{f \in$ $\left.G_{p_{0}} \mid f_{*} T_{p_{0}} N_{0}=T_{p_{0}} N_{0}\right\}$.

Proof. (a) follows directly from the fact that $G_{p_{0}}$ acts transitively on $\mathfrak{F}_{p_{0}}\left(N_{0}, M\right)$ and its isotropy group at $N_{0}$ is $G_{p_{0}} \cap K$. In the situation of (b), we have to show $G_{p_{0}} \cap K=H:=\left\{f \in G_{p_{0}} \mid f_{*} T_{p_{0}} N_{0}=T_{p_{0}} N_{0}\right\}$. Here, $G_{p_{0}} \cap K \subset H$ is in fact true without the hypothesis that $N_{0}$ is totally geodesic in $M$, because $N_{0}$ is a regular submanifold of $M . G_{p_{0}} \cap K \supset H$ follows from the rigidity of totally geodesic submanifolds.

THEOREM 3. The codimension of $\mathfrak{F}_{p_{0}}\left(N_{0}, M\right)$ in $\mathfrak{F}\left(N_{0}, M\right)$ is equal to the codimension of $N_{0}$ in $M$.

Proof. Consider $P:=M \times \mathfrak{F}\left(N_{0}, M\right)$. The incidence set $\mathbb{X}:=\{(p, N) \in P \mid p \in N\}$ is the orbit through $\left(p_{0}, N_{0}\right)$ of the action

$$
G \times P \rightarrow P,(f,(p, N)) \mapsto(f(p), f(N))
$$

and therefore a submanifold of $P$. The canonical projections

$$
\varphi_{1}: \mathbb{X} \rightarrow M \quad \text { resp. } \quad \varphi_{2}: \mathbb{X} \rightarrow \mathfrak{F}\left(N_{0}, M\right)
$$

describe fibre bundles with typical fibre

$$
\mathfrak{F}_{p_{0}}\left(N_{0}, M\right) \quad \text { resp. } \quad N_{0}
$$

associated to the principal fibre bundles

$$
\sigma_{1}: G \rightarrow M, f \mapsto f\left(p_{0}\right) \quad \text { resp. } \quad \sigma_{2}: G \rightarrow \mathfrak{F}\left(N_{0}, M\right), f \mapsto f\left(N_{0}\right)
$$

with structure group $G_{p_{0}}$ resp. $K$ via the association maps

$$
\begin{aligned}
& \varrho_{1}: G \times \mathfrak{F}_{p_{0}}\left(N_{0}, M\right) \rightarrow \mathbb{X},(f, N) \mapsto\left(f\left(p_{0}\right), f(N)\right) \quad \text { resp. } \\
& \varrho_{2}: G \times N_{0} \rightarrow \mathbb{X},(f, p) \mapsto\left(f(p), f\left(N_{0}\right)\right) ;
\end{aligned}
$$

see $[\mathrm{B}], \S 6.5$. Thus, we have two ways to calculate $\operatorname{dim} \mathbb{X}$ :

$$
\begin{aligned}
\operatorname{dim} \mathbb{X} & =\operatorname{dim} M+\operatorname{dim} \mathfrak{F}_{p_{0}}\left(N_{0}, M\right) \\
& =\operatorname{dim} \mathfrak{F}\left(N_{0}, M\right)+\operatorname{dim} N_{0} .
\end{aligned}
$$

Therefore, the theorem is proved.

1.3. A naturally reductive structure on $\mathfrak{F}\left(N_{0}, M\right)$. In the previous situation we denote the Lie algebras of $G$ and $K$ by $\mathfrak{g}$ and $\mathfrak{k}$, respectively, but suppose that $M$ is a Riemannian symmetric space of compact type, meaning in particular that the Killing form $\beta$ of $\mathfrak{g}$ is negative definite.

Proposition 4. In this situation, $-\beta$ induces a G-invariant Riemannian metric on $\mathfrak{F}\left(N_{0}, M\right)$, and the subspace $\mathfrak{m}:=\{X \in \mathfrak{g} \mid \forall Y \in \mathfrak{k}: \beta(X, Y)=0\}$ defines a naturally reductive structure on $\mathfrak{F}\left(N_{0}, M\right)$ (see Remark 3 below).

Proof. As $\beta$ is $\operatorname{Ad}(G)$-invariant, and $\mathfrak{k}, \mathfrak{m}$ are $\operatorname{Ad}(K)$-invariant subspaces of $\mathfrak{g}$ satisfying $\mathfrak{g}=\mathfrak{k} \oplus \mathfrak{m}$, it follows that $\mathfrak{m}$ defines a reductive structure on $\mathfrak{F}\left(N_{0}, M\right)$, see [KN], p. 190 . Furthermore, $-\beta \mid(\mathfrak{m} \times \mathfrak{m})$ induces a $G$-invariant metric on $\mathfrak{F}\left(N_{0}, M\right)$, whereby $\mathfrak{F}\left(N_{0}, M\right)$ becomes a Riemannian homogeneous $G$-space. 
Because $\mathfrak{k}$ and $\mathfrak{m}$ are orthogonal with respect to $\beta$, we have

$$
\forall X, Y, Z \in \mathfrak{m}: \beta\left([Z, X]_{\mathfrak{m}}, Y\right)+\beta\left(X,[Z, Y]_{\mathfrak{m}}\right)=0,
$$

where $X_{\mathfrak{m}}$ denotes the projection of $X \in \mathfrak{g}$ onto $\mathfrak{m}$ along $\mathfrak{k}$. Therefore $\mathfrak{F}\left(N_{0}, M\right)$ is naturally reductive; see $[\mathrm{KN}]$, Theorem X.3.3(2), p. 201.

REMARK 1. In general, $\mathfrak{F}\left(N_{0}, M\right)$ does not become a symmetric space in this way, as the examples of Sections 2.2 and 3.2 will show.

REMARK 2. For the studies of specific examples of congruence manifolds in the following sections, it is more convenient to replace in the constructions of the present section the group $G=I(M)$ acting on $\mathfrak{F}\left(N_{0}, M\right)$ by a linear group $\widetilde{G}$ which is a covering group of an open subgroup $G^{\prime}$ of $G$ that still acts transitively on $\mathfrak{F}\left(N_{0}, M\right)$. In fact, with only one exception (see Theorem $8(\mathrm{c})$ ), the families we study are connected; then $\widetilde{G}$ will be chosen as a covering group of the neutral component $G_{0}$. Because the covering map $\tau: \widetilde{G} \rightarrow G^{\prime}$ gives rise to an isomorphism between the Lie algebras of $\widetilde{G}$ and $G$, we obtain an isomorphic naturally reductive structure in this way.

We now describe the construction with respect to $\widetilde{G}$ more formally: The map $\Psi:=$ $\left(G^{\prime} \hookrightarrow G\right) \circ \tau: \widetilde{G} \rightarrow G$ is a homomorphism of Lie groups, and its linearization $\Psi_{L}: \widetilde{\mathfrak{g}} \rightarrow \mathfrak{g}$ is an isomorphism of Lie algebras; here $\widetilde{\mathfrak{g}}$ is the Lie algebra of $\widetilde{G}$. Therefore the Killing form $\widetilde{\beta}$ of $\widetilde{\mathfrak{g}}$ is connected to $\beta$ by $\widetilde{\beta}(\widetilde{X}, \widetilde{Y})=\beta\left(\Psi_{L}(\widetilde{X}), \Psi_{L}(\widetilde{Y})\right)$.

Of course, $\widetilde{G}$ still acts transitively on $\mathfrak{F}\left(N_{0}, M\right)$ via the action $(\widetilde{g}, N) \mapsto$ $(\Psi(\widetilde{g}))(N)$, and its isotropy group at $N_{0}$ is $\widetilde{K}:=\Psi^{-1}\left(K \cap G^{\prime}\right)$; so $\mathfrak{F}\left(N_{0}, M\right)$ is isomorphic to the homogeneous space $\widetilde{G} / \widetilde{K}$. Moreover, denoting the Lie algebra of $\widetilde{K}$ by $\widetilde{\mathfrak{k}}$, we have $\widetilde{\mathfrak{g}}=\widetilde{\mathfrak{k}} \oplus \widetilde{\mathfrak{m}}$ with $\widetilde{\mathfrak{m}}:=\Psi_{L}^{-1}(\mathfrak{m})=\{\widetilde{X} \in \widetilde{\mathfrak{g}} \mid \forall \widetilde{Y} \in \widetilde{\mathfrak{k}}: \widetilde{\beta}(\widetilde{X}, \widetilde{Y})=0\} ;$ and because we have $\Psi_{L} \circ \operatorname{Ad}_{\widetilde{G}}(\widetilde{g})=\operatorname{Ad}_{G}(\Psi(\widetilde{g})) \circ \Psi_{L}$ for every $\widetilde{g} \in \widetilde{G}, \widetilde{\mathfrak{m}}$ is $\operatorname{Ad}(\widetilde{K})$-invariant and therefore defines a naturally reductive structure on the homogeneous $\widetilde{G}$-space $\mathfrak{F}\left(N_{0}, M\right)$ and $\widetilde{\beta}$ induces the $\widetilde{G}$-invariant Riemannian metric on $\mathfrak{F}\left(N_{0}, M\right)$ also defined by $\beta$. Thus, this naturally reductive homogeneous $\widetilde{G}$-space structure on $\mathfrak{F}\left(N_{0}, M\right)$ is isomorphic to the naturally reductive homogeneous $G$-space structure constructed in Proposition 4, and all results of the present section remain true if we replace $G$ by $\widetilde{G}$, and the actions of $G$ on $M$ and on $\mathfrak{F}\left(N_{0}, M\right)$ by the actions of $\widetilde{G}$ on these spaces.

REMARK 3 (On reductive homogeneous spaces). Suppose $M$ is a reductive homogeneous $G$-space; we fix some $p \in M$ and denote the action of $G$ on $M$ by $\varphi: G \times M \rightarrow M$ and the reductive structure of $M$ (at $p)$ by $\mathfrak{m}$; we interpret the elements of $\mathfrak{m}$ as left-invariant vector fields on $G$ and define $\varphi^{p}: G \rightarrow M, g \mapsto \varphi(g, p)$. According to Nomizu, $\mathfrak{m}$ induces two covariant derivatives on $M$ of particular importance: the torsion-free canonical derivative of the first kind $\nabla$ (see [No], Theorem 10.1), characterized by

$$
\forall X, Y \in \mathfrak{m}: \nabla_{X} \varphi_{*}^{p} Y=\frac{1}{2} \cdot \varphi_{*}^{p}[X, Y]
$$

and the canonical derivative of the second kind $\nabla^{0}$ (see [No], Theorem 10.2), characterized by

$$
\forall X, Y \in \mathfrak{m}: \nabla_{X}^{0} \varphi_{*}^{p} Y \equiv 0 .
$$

For every $X \in \mathfrak{m}$ we denote by $\gamma_{X}: \mathbb{R} \rightarrow G$ the 1-parameter-subgroup of $G$ induced by $X$. Then $\varphi^{p} \circ \gamma_{X}: \mathbb{R} \rightarrow M$ is a geodesic with respect to $\nabla^{0}$, and every $\nabla^{0}$-geodesic $\gamma$ 
of $M$ with $\gamma(0)=p$ can be obtained in this way (see [KN], Corollary X.2.5, p. 192). As the difference tensor $\nabla-\nabla^{0}$ is skew-symmetric, the $\nabla^{0}$-geodesics and the $\nabla$-geodesics in $M$ coincide.

If, in this setting, $M$ is equipped with a Riemannian metric such that $G$ acts by isometries on $M, M$ is called naturally reductive if $\nabla$ coincides with the Levi-Civita derivative induced by the Riemannian metric on $M$.

A further reductive homogeneous space $M^{\prime}$ (with group action $\varphi^{\prime}: G^{\prime} \times M^{\prime} \rightarrow M^{\prime}$ and reductive structure $\mathfrak{m}^{\prime}$ ) is called a reductive homogeneous subspace of $M$ if $M^{\prime}$ is a submanifold of $M, G^{\prime}$ is a subgroup of $G, \varphi^{\prime}=\varphi \mid\left(G^{\prime} \times M^{\prime}\right)$ and $\mathfrak{m}^{\prime} \subset \mathfrak{m}$. In this situation, $M^{\prime}$ necessarily is a totally geodesic submanifold of $M$ (with respect to either of the canonical derivatives of $M$ resp. $M^{\prime}$ ), as the following argument shows: If $\gamma$ is a geodesic of $M^{\prime}$ with $\gamma(0)=p \in M^{\prime}$, we have $\gamma=\left(\varphi^{\prime}\right)^{p} \circ \gamma_{X}$ with $X \in \mathfrak{m}^{\prime}$; because of $\mathfrak{m}^{\prime} \subset \mathfrak{m}, \gamma$ also is a geodesic of $M$.

Finally, we mention that if $M$ is a naturally reductive homogeneous space, we call a reductive homogeneous subspace $M^{\prime}$ of $M$ which carries the structure of a naturally reductive homogeneous space a naturally reductive homogeneous subspace if the inclusion map $M^{\prime} \hookrightarrow M$ is a homothetic immersion, i.e. if there is a constant $c \in \mathbb{R}_{+}$so that for every $p \in M^{\prime}, v, w \in T_{p} M^{\prime}$ we have $\langle v, w\rangle_{M^{\prime}}=c \cdot\langle v, w\rangle_{M}$. (Such a factor occurs necessarily in Theorem 7.)

2. Congruence families in the complex projective space. From the standpoint of algebraic geometry, the simplest submanifolds of the complex projective space are those defined by linear equations, namely the projective subspaces, and those defined by quadratic equations, namely the complex quadrics. In this section, we investigate the congruence families induced in a complex projective space by these submanifolds.

We fix some notations: Let $\mathbb{V}$ be a unitary vector space of complex dimension $n+1 \geq 2$. We denote the complex structure $v \mapsto i \cdot v$ of $\mathbb{V}$ by $J$, and the complex inner product of $\mathbb{V}$ by $\langle\cdot, \cdot\rangle_{\mathbb{C}}$. The latter induces the real inner product $\langle\cdot, \cdot\rangle:=\operatorname{Re}\langle\cdot, \cdot\rangle_{\mathbb{C}}$ and thereby the norm $\|v\|$. For every $k \in\{1, \ldots, n\}$, let $G_{k}(\mathbb{V})$ denote the Grassmannian manifold of $k$-dimensional complex subspaces of $\mathbb{V}$, which is known to be a Hermitian symmetric space of type Alll (see $[\mathrm{H}]$, p. 518 ), and for every $V \in G_{k}(\mathbb{V})$, we put $S(V):=\{v \in V \mid\|v\|=1\}$. By $\mathbb{P}(\mathbb{V})$ we denote the complex projective space $G_{1}(\mathbb{V})$ and by $\pi: S(\mathbb{V}) \rightarrow \mathbb{P}(\mathbb{V}), v \mapsto[v]$ the Hopf fibration. We always view $\mathbb{P}(\mathbb{V})$ as a Kähler manifold, so that $\pi$ becomes a Hermitian submersion (see [KN], Example XI.10.5, p. 273f.). For every $V \in G_{k+1}(\mathbb{V})$, we put $[V]:=\{\pi(v) \mid v \in S(V)\}$, this being a $k$-dimensional projective subspace in $\mathbb{P}(\mathbb{V})$. If, on the other hand, $\Lambda$ is a $k$-dimensional projective subspace of $\mathbb{P}(\mathbb{V})$, then $\widehat{\Lambda}:=\left\{\lambda \cdot v \mid \lambda \in \mathbb{R}, v \in \pi^{-1}(\Lambda)\right\}$ is an element of $G_{k+1}(\mathbb{V})$.

For any unitary transformation $B \in \mathrm{U}(\mathbb{V})$, there is exactly one holomorphic isometry $\underline{B}: \mathbb{P}(\mathbb{V}) \rightarrow \mathbb{P}(\mathbb{V})$ such that $\underline{B} \circ \pi=\pi \circ B \mid S(\mathbb{V})$, and any holomorphic isometry of $\mathbb{P}(\mathbb{V})$ is obtained in this way. If $G$ is a subgroup of $\mathrm{U}(\mathbb{V})$, we put $\underline{G}:=\{\underline{B} \mid B \in G\}$; this is a subgroup of the group $I_{h}(\mathbb{P}(\mathbb{V}))$ of all holomorphic isometries of $\mathbb{P}(\mathbb{V})$. In fact, we have $I_{h}(\mathbb{P}(\mathbb{V}))=I(\mathbb{P}(\mathbb{V}))_{0}=\mathrm{SU}(\mathbb{V})$. For the following considerations of congruence families $\mathfrak{F}\left(N_{0}, \mathbb{P}(\mathbb{V})\right)$ we will use the $(n+1)$-fold covering $\tau: \mathrm{SU}(\mathbb{V}) \rightarrow I_{h}(\mathbb{P}(\mathbb{V})), B \mapsto \underline{B}$, applying 
Remark 2. This means, we consider these families as naturally reductive homogeneous $\mathrm{SU}(\mathbb{V})$-spaces.

2.1. Projective subspaces in $\mathbb{P}(\mathbb{V})$. We fix some $k \leq n$. The set of $k$-dimensional projective subspaces of $\mathbb{P}(\mathbb{V})$ forms a congruence family in $\mathbb{P}(\mathbb{V})$, which we denote by $\mathfrak{F}\left(\mathbb{P}^{k}, \mathbb{P}(\mathbb{V})\right.$ ). Because $\mathrm{SU}(\mathbb{V})$ acts transitively on $\mathfrak{F}\left(\mathbb{P}^{k}, \mathbb{P}(\mathbb{V})\right)$ via the action $(B, \Lambda) \mapsto \underline{B}(\Lambda)$, we may consider $\mathfrak{F}\left(\mathbb{P}^{k}, \mathbb{P}(\mathbb{V})\right)$ as a naturally reductive homogeneous $\mathrm{SU}(\mathbb{V})$-space as explained above.

TheOREM 5. $\mathfrak{F}\left(\mathbb{P}^{k}, \mathbb{P}(\mathbb{V})\right)$ may be equipped with the structure of a Hermitian symmetric space isomorphic to $G_{k+1}(\mathbb{V})$, and this structure is compatible with the naturally reductive structure of $\mathfrak{F}\left(\mathbb{P}^{k}, \mathbb{P}(\mathbb{V})\right)$ introduced before. Then, for any $p \in \mathbb{P}(\mathbb{V}), \mathfrak{F}_{p}\left(\mathbb{P}^{k}, \mathbb{P}(\mathbb{V})\right)$ is a Hermitian symmetric subspace (in particular a complex submanifold) of $\mathfrak{F}\left(\mathbb{P}^{k}, \mathbb{P}(\mathbb{V})\right.$ ) isomorphic to the Grassmannian $G_{k}\left(p^{\perp}\right)$.

Proof. The first part of the theorem follows from the fact that

$$
\theta: G_{k+1}(\mathbb{V}) \rightarrow \mathfrak{F}\left(\mathbb{P}^{k}, \mathbb{P}(\mathbb{V})\right), V \mapsto[V]
$$

is an isomorphism of naturally reductive $\mathrm{SU}(\mathbb{V})$-spaces.

For the second part, we consider the embedding $f: G_{k}\left(p^{\perp}\right) \rightarrow G_{k+1}(\mathbb{V}), W \mapsto$ $W \oplus p$ and the injective Lie group homomorphism $F: \mathrm{SU}\left(p^{\perp}\right) \rightarrow \mathrm{SU}(\mathbb{V})$ characterized by $F(B) \mid p=\operatorname{id}_{p}$ and $F(B) \mid p^{\perp}=B$ for all $B \in \mathrm{SU}\left(p^{\perp}\right)$. Then the pair $(f, F)$ is a homomorphism of Hermitian symmetric spaces ${ }^{3}$, and we have $F\left(\mathrm{SU}\left(p^{\perp}\right)\right)=\{B \in$ $\left.\mathrm{SU}(\mathbb{V})|B| p=\mathrm{id}_{p}\right\}=: \mathrm{SU}(\mathbb{V})_{p}$. As $f\left(G_{k}\left(p^{\perp}\right)\right)$ is mapped onto $\mathfrak{F}_{p}\left(\mathbb{P}^{k}, \mathbb{P}(\mathbb{V})\right)$ by $\theta$, we see that $\mathfrak{F}_{p}\left(\mathbb{P}^{k}, \mathbb{P}(\mathbb{V})\right)$ is a Hermitian symmetric $\mathrm{SU}(\mathbb{V})_{p}$-subspace of the Hermitian symmetric $\mathrm{SU}(\mathbb{V})$-space $\mathfrak{F}\left(\mathbb{P}^{k}, \mathbb{P}(\mathbb{V})\right)$.

2.2. Complex quadrics in $\mathbb{P}(\mathbb{V})$. In algebraic geometry, a complex quadric in $\mathbb{P}(\mathbb{V})$ simply is a subvariety of $\mathbb{P}(\mathbb{V})$ defined by a non-degenerate quadratic form on some linear subspace of $\mathbb{V}$. However, the set of such quadrics forms an infinite multitude of congruence families. For this reason, we require a binding to the Riemannian geometry of $\mathbb{P}(\mathbb{V})$ and consider only those quadrics which are extrinsically symmetric submanifolds of $\mathbb{P}(\mathbb{V})$; see [NT], p. 171.

To describe them explicitly, we need the concept of a conjugation: We call an antilinear ${ }^{4}$, self-adjoint map $A: \mathbb{V} \rightarrow \mathbb{V}$ a partial conjugation if $A \mid A(\mathbb{V})$ is an orthogonal map on $A(\mathbb{V})$. The (real) rank of a partial conjugation is necessarily even, and we denote the set of partial conjugations on $\mathbb{V}$ which are of $\operatorname{rank} 2 \ell$ by $\operatorname{Con}_{\ell}(\mathbb{V})$. The elements of $\operatorname{Con}_{n+1}(\mathbb{V})$ are called full conjugations.

\footnotetext{
${ }^{3}$ If $M=G / K, M^{\prime}=G^{\prime} / K^{\prime}$ are symmetric spaces and $\sigma: G \rightarrow G$ and $\sigma^{\prime}: G^{\prime} \rightarrow G^{\prime}$ the involutive automorphisms that define them, we call a homomorphism (isomorphism) of reductive homogeneous spaces $(f, F)$ between $M$ and $M^{\prime}$ a homomorphism (isomorphism) of symmetric spaces if $F \circ \sigma=\sigma^{\prime} \circ F$. If $M$ and $M^{\prime}$ are Hermitian symmetric and $f$ is holomorphic, we speak of a homomorphism (isomorphism) of Hermitian symmetric spaces. In the case $G=G^{\prime}, F=\mathrm{id}_{G}$, we call $f$ simply a homomorphism (isomorphism) of symmetric spaces.

${ }^{4}$ We call an $\mathbb{R}$-linear map $L: V \rightarrow W$ between the complex vector spaces $V$ and $W$ antilinear, if $\forall \lambda \in \mathbb{C}, v \in V: L(\lambda v)=\bar{\lambda} \cdot L v$ holds.
} 
Every $A \in \operatorname{Con}_{\ell}(\mathbb{V})$ is real diagonalizable; the only possible eigenvalues of $A$ are $-1,0,1$, and the eigenvalue 0 occurs if and only if $A$ is not full. The eigenspaces $\operatorname{Eig}(A, \lambda)$ $(\lambda \in\{-1,0,1\})$ are pairwise orthogonal, $\operatorname{ker} A=\operatorname{Eig}(A, 0)$ is a complex linear subspace of $\mathbb{V}, V(A):=\operatorname{Eig}(A, 1)$ is a totally real subspace of $\mathbb{V}$, and $J V(A)=\operatorname{Eig}(A,-1)$. Thus, we have the decomposition $\mathbb{V}=V(A) \oplus J V(A) \oplus \operatorname{ker} A$.

We now fix $k \leq n-1$ and obtain for each $A \in \operatorname{Con}_{k+2}(\mathbb{V})$ the quadric

$$
Q(A, \mathbb{V}):=\left\{\pi(v) \mid v \in S(A(\mathbb{V})),\langle v, A v\rangle_{\mathbb{C}}=0\right\},
$$

a $k$-dimensional complex submanifold of $\mathbb{P}(\mathbb{V})$ lying in the projective subspace $[A(\mathbb{V})]$. The set of these quadrics forms a congruence family $\mathfrak{F}\left(Q^{k}, \mathbb{P}(\mathbb{V})\right)$, and it can be shown that they are exactly the extrinsically symmetric submanifolds mentioned above. We will call these quadrics simply complex quadrics in the sequel. If $A$ is full, $Q(A, \mathbb{V})$ is a complex hypersurface in $\mathbb{P}(\mathbb{V})$.

The ordinary example is the quadric $\left\{\pi(z) \mid z \in S\left(\mathbb{C}^{n+1}\right), \sum_{\nu=1}^{n+1} z_{\nu}^{2}=0\right\}$ of $\mathbb{P}\left(\mathbb{C}^{n+1}\right)$ defined by the full conjugation $A_{0}: \mathbb{C}^{n+1} \rightarrow \mathbb{C}^{n+1}, z \mapsto \bar{z}$.

TheOrem 6. For $k \leq n-1$ we have:

(a) $\mathfrak{F}\left(Q^{k}, \mathbb{P}(\mathbb{V})\right)$ is a naturally reductive homogeneous $\mathrm{SU}(\mathbb{V})$-space (in the way described in Proposition 4 and Remark 2) of dimension $2 n(k+2)-\frac{1}{2} \cdot(3 k+4)(k+1)$. $\mathfrak{F}_{p}\left(Q^{k}, \mathbb{P}(\mathbb{V})\right)$ is a homogeneous subspace of $\mathfrak{F}\left(Q^{k}, \mathbb{P}(\mathbb{V})\right)$ by Proposition $1(b)$; its codimension in $\mathfrak{F}\left(Q^{k}, \mathbb{P}(\mathbb{V})\right)$ is $2(n-k)$.

(b) (i) In the case $k<n-1$, the naturally reductive structure on $\mathfrak{F}\left(Q^{k}, \mathbb{P}(\mathbb{V})\right)$ is not induced by a symmetric structure.

(ii) In the case $k=n-1$, the naturally reductive structure on $\mathfrak{F}\left(Q^{n-1}, \mathbb{P}(\mathbb{V})\right)$ is induced by a symmetric structure, and in this way $\mathfrak{F}\left(Q^{n-1}, \mathbb{P}(\mathbb{V})\right)$ is an irreducible Riemannian symmetric space of type Al; this means that its universal cover is isomorphic to $\mathrm{SU}(n+1) / \mathrm{SO}(n+1)$, see also $[H]$, p. 518 .

Proof. (a) For any $A \in \operatorname{Con}_{k+2}(\mathbb{V})$ and $B \in \mathrm{U}(\mathbb{V})$, we have $B A B^{-1} \in \operatorname{Con}_{k+2}(\mathbb{V})$ and $\underline{B}(Q(A, \mathbb{V}))=Q\left(B A B^{-1}, \mathbb{V}\right)$; thus $\mathrm{U}(\mathbb{V})$ acts transitively on the space $\mathfrak{F}\left(Q^{k}, \mathbb{P}(\mathbb{V})\right)$ via the action $(B, Q) \mapsto \underline{B}(Q)$. For any $\lambda \in S^{1}$, we have $\underline{\lambda B}=\underline{B}$, and therefore already $\mathrm{SU}(\mathbb{V})$ acts transitively on $\mathfrak{F}\left(Q^{k}, \mathbb{P}(\mathbb{V})\right)$. Hence, we may consider $\mathfrak{F}\left(Q^{k}, \mathbb{P}(\mathbb{V})\right)$ as a naturally reductive homogeneous $\mathrm{SU}(\mathbb{V})$-space via Proposition 4 and Remark 2.

To calculate the isotropy group $K$ of the action of $\mathrm{SU}(\mathbb{V})$ on $\mathfrak{F}\left(Q^{k}, \mathbb{P}(\mathbb{V})\right)$ at $Q(A, \mathbb{V})$ (for some $A \in \operatorname{Con}_{k+2}(\mathbb{V})$ ), one has to notice that, for any $A, A^{\prime} \in \operatorname{Con}_{k+2}(\mathbb{V})$, we have $Q(A, \mathbb{V})=Q\left(A^{\prime}, \mathbb{V}\right)$ if and only if there exists $\lambda \in S^{1}$ so that $A^{\prime}=\lambda A$. It follows that

$$
K=\left\{B \in \mathrm{SU}(\mathbb{V}) \mid \exists \lambda \in S^{1}: \lambda B(V(A))=V(A)\right\} .
$$

From $\lambda B(V(A))=V(A)$ one derives $(\lambda B) \mid V(A) \in \mathrm{O}(V(A))$ and $(\lambda B) \mid(\operatorname{ker} A) \in \mathrm{U}(\operatorname{ker} A)$, and these conditions permit one to calculate $\operatorname{dim} K=\operatorname{dim} \mathrm{O}(V(A))+\operatorname{dim} \mathrm{U}(\operatorname{ker} A)$; note that $\lambda$ can attain only discrete values. Now $\operatorname{dim} \mathfrak{F}\left(Q^{k}, \mathbb{P}(\mathbb{V})\right)=\operatorname{dim} \mathrm{SU}(\mathbb{V})-\operatorname{dim} K \operatorname{can}$ be calculated easily, and the codimension of $\mathfrak{F}_{p}\left(Q^{k}, \mathbb{P}(\mathbb{V})\right)$ is derived from Theorem 3 .

(b)(i) We denote the reductive structure of $\mathfrak{F}\left(Q^{k}, \mathbb{P}(\mathbb{V})\right) \cong \mathrm{SU}(\mathbb{V}) / K$ by $\mathfrak{m}$, and the Lie algebra of $K$ by $\mathfrak{k}$. 
In block matrix notation with respect to the decomposition $\mathbb{V}=\mathbb{C} \cdot V(A) \oplus$ ker $A$, we have

$$
\mathfrak{k}=\left\{\left(\begin{array}{cc}
X+\alpha J & 0 \\
0 & Y+\alpha J
\end{array}\right) \mid \begin{array}{c}
X \in \mathfrak{o}(V(A)), Y \in \mathfrak{u}(\operatorname{ker} A) \\
\alpha=-\frac{1}{i(n+1)} \operatorname{tr}_{\mathbb{C}} Y
\end{array}\right\} \subset \mathfrak{s u}(\mathbb{V}) .
$$

Note that any endomorphism $B: V(A) \rightarrow V(A)$ may be extended by the definition $B(x+J y):=B x+J(B y)$ for $x, y \in V(A)$ to an endomorphism on $\mathbb{C} \cdot V(A)$ (we will call this process complexification in the sequel), and if $B$ is skew-adjoint, its complexification is skew-Hermitian; in the previous equation, we interpreted the elements of $\mathfrak{o}(V(A))$ in this way. Further, we have $\mathfrak{m}=\mathfrak{m}_{1} \oplus \mathfrak{m}_{2}$, where

$$
\begin{aligned}
\mathfrak{m}_{1} & =\left\{\left(\begin{array}{cc}
0 & -Z^{*} \\
Z & 0
\end{array}\right) \mid Z: \mathbb{C} \cdot V(A) \rightarrow \operatorname{ker} A \text { complex-linear }\right\} \\
\text { and } \quad \mathfrak{m}_{2} & =\left\{\left(\begin{array}{cc}
J X & 0 \\
0 & 0
\end{array}\right) \mid X \in \operatorname{End}_{\text {self-adjoint }}(V(A)), \operatorname{tr} X=0\right\} .
\end{aligned}
$$

Now, choose an orthonormal basis $\left(a_{1}, \ldots, a_{k+2}\right)$ of $V(A)$. Then, $\left(a_{1}, \ldots, a_{k+2}\right)$ also is a unitary basis of $\mathbb{C} \cdot V(A)$, which we expand to a unitary basis $\left(a_{1}, \ldots, a_{n+1}\right)$ of $\mathbb{V}$. Consider the endomorphisms $D, E: \mathbb{V} \rightarrow \mathbb{V}$ given by

$$
D a_{1}=i a_{1}, D a_{2}=-i a_{2}, D a_{j}=0 \text { for } j \geq 3
$$

and

$$
E a_{1}=a_{k+3}, E a_{k+3}=-a_{1}, E a_{j}=0 \text { for } j \in\{2, \ldots, n+1\} \backslash\{k+3\},
$$

respectively. We have $D \in \mathfrak{m}_{2} \subset \mathfrak{m}$ and $E \in \mathfrak{m}_{1} \subset \mathfrak{m}$. However, a simple calculation shows $[D, E] a_{1}=-i a_{k+3}$ and therefore $[D, E] \notin \mathfrak{k}$. Thus, we have $[\mathfrak{m}, \mathfrak{m}] \not \subset \mathfrak{k}$, showing that $\mathfrak{m}$ cannot be induced by a symmetric structure on $\mathfrak{F}\left(Q^{k}, \mathbb{P}(\mathbb{V})\right)$.

(b)(ii) In the case $k=n-1$, we have $\operatorname{ker} A=\{0\}$ and therefore we see from equation

$$
K=\left\{\lambda B \in \mathrm{SU}(\mathbb{V}) \mid B \in \mathrm{SO}(V(A)), \lambda \in S^{1}, \lambda^{n+1}=1\right\} .
$$

But now we consider $\mathfrak{F}\left(Q^{k}, \mathbb{P}(\mathbb{V})\right)$ as an $I_{h}(\mathbb{P}(\mathbb{V}))$-space. $I_{h}(\mathbb{P}(\mathbb{V}))$ also acts transitively on $\mathfrak{F}\left(Q^{k}, \mathbb{P}(\mathbb{V})\right)$ and we see from (4) that the isotropy group of this action at the "point" $Q(A, \mathbb{V})$ is $\mathrm{SO}(V(A))$. Because $A$ is a full conjugation on $\mathbb{V}, \underline{A}$ is an anti-holomorphic

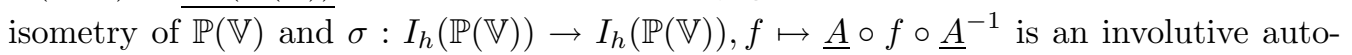
morphism of Lie groups with $\operatorname{Fix}(\sigma)=\mathrm{SO}(V(A))$. Therefore, $\left(I_{h}(\mathbb{P}(\mathbb{V})), \mathrm{SO}(V(A)), \sigma\right)$ is

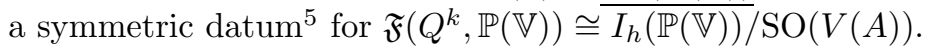

The $(n+1)$-fold covering $\tau: \mathrm{SU}(\mathbb{V}) \rightarrow I_{h} \overline{(\mathbb{P}(\mathbb{V})), B} \mapsto \underline{B}$ induces the covering $\mathrm{SU}(\mathbb{V}) / \mathrm{SO}(V(A)) \rightarrow \mathfrak{F}\left(Q^{k}, \mathbb{P}(\mathbb{V})\right), B \cdot \mathrm{SO}(V(A)) \mapsto Q\left(B A B^{-1}, \mathbb{V}\right)$.

If we identify the Lie algebra of $I_{h}(\mathbb{P}(\mathbb{V}))$ with $\mathfrak{s u}(\mathbb{V})$ via the isomorphism $\tau_{L}, \sigma$ induces also on the $\mathrm{SU}(\mathbb{V})$-space $\mathfrak{F}\left(Q^{k}, \mathbb{P}(\mathbb{V})\right)$ a reductive structure $\mathfrak{m}_{\sigma}:=\left\{X \in \mathfrak{s u}(\mathbb{V}) \mid \sigma_{L}(X)=\right.$ $-X\}$. It remains to show that $\mathfrak{m}_{\sigma}$ is equal to the original naturally reductive structure $\mathfrak{m}$ on $\mathfrak{F}\left(Q^{k}, \mathbb{P}(\mathbb{V})\right)$. For this we note that the Killing form of $\mathfrak{s u}(\mathbb{V})$ is invariant under the automorphism $\sigma_{L}$ of $\mathfrak{s u}(\mathbb{V})$. A simple calculation now shows $\mathfrak{m}_{\sigma}=\mathfrak{m}$; see $[\mathrm{KN}]$, Lemma p. 233 .

\footnotetext{
${ }^{5} \mathrm{~A}$ symmetric space in the terminology of [KN], p. 225.
} 
3. Congruence families in a complex quadric. Continuing to use the notations of Section 2, we fix some full conjugation $A_{0} \in \mathrm{Con}_{n+1}(\mathbb{V})$ and consider the complex hypersurface $Q:=Q\left(A_{0}, \mathbb{V}\right)$ in $\mathbb{P}(\mathbb{V})$. $Q$ now plays the role of the ambient space, in which we will study congruence families induced by projective subspaces or complex quadrics contained in $Q$. These families will turn out to be nice submanifolds of $\mathfrak{F}\left(\mathbb{P}^{k}, \mathbb{P}(\mathbb{V})\right)$ and $\mathfrak{F}\left(Q^{k}, \mathbb{P}(\mathbb{V})\right)$, respectively. We put $m:=\operatorname{dim}_{\mathbb{C}} Q=n-1$.

Any $B \in \mathrm{O}\left(V\left(A_{0}\right)\right)$ may be interpreted via complexification as an element of $\mathrm{U}(\mathbb{V})$, and an easy calculation shows that in this situation, the holomorphic isometry $\underline{B}$ leaves $Q$ invariant, and therefore $\underline{B} \mid Q$ is a holomorphic isometry of $Q$. Similarly, $\underline{A_{0} \circ B} \mid Q$ is an anti-holomorphic ${ }^{6}$ isometry of $Q$. It is, however, a non-trivial fact that every holomorphic resp. anti-holomorphic isometry of $Q$ is obtained in this manner, and that for $m \geq 3$, any isometry of $Q$ is either holomorphic or anti-holomorphic; see [Re], Corollary 2. It follows that $Q$ is a Hermitian symmetric space $\cong \mathrm{SO}(m+2) /(\mathrm{SO}(2) \times \mathrm{SO}(m))$. It can be shown that $Q$ is of rank 2 for $m \geq 2$ and irreducible for $m \neq 2$.

The map $\tau: \mathrm{O}\left(V\left(A_{0}\right)\right) \rightarrow I_{h}(Q), B \mapsto \underline{B} \mid Q$ is a two-fold group covering with kernel $\{ \pm$ id $\}$; here $I_{h}(Q)$ denotes the group of holomorphic isometries on $Q$. Therefore, $I_{h}(Q)$ is connected if $m$ is odd, and has exactly two connected components if $m$ is even. Further, we have $I(Q)_{0}=\left\{\underline{B}|Q| B \in \mathrm{SO}\left(V\left(A_{0}\right)\right)\right\}$. In the sequel, we will apply Remark 2 using either $\tau$ or $\tau \mid \mathrm{SO}\left(V\left(A_{0}\right)\right)$ to view the congruence families $\mathfrak{F}\left(N_{0}, Q\right)$ as naturally reductive homogeneous $\mathrm{O}\left(V\left(A_{0}\right)\right)$-spaces or $\mathrm{SO}\left(V\left(A_{0}\right)\right)$-spaces.

As we already mentioned in the proof of Theorem $6(\mathrm{a}), A^{\prime}, A^{\prime \prime} \in \mathrm{Con}_{k+2}(\mathbb{V})$ describe the same quadric if and only if we have $A^{\prime \prime}=\lambda \cdot A^{\prime}$ for some $\lambda \in S^{1}$. Therefore, there is a one-to-one-correspondence between $k$-dimensional complex quadrics in $\mathbb{P}(\mathbb{V})$ and circles $\left\{\lambda A^{\prime} \mid \lambda \in S^{1}\right\} \subset \operatorname{Con}_{k+2}(\mathbb{V})$.

3.1. Complex quadrics in $Q$. Suppose $m \geq 3 ;^{7}$ fix some integer $k<m$. In this section, we study $k$-dimensional complex quadrics contained in $Q$. As it should be emphasized, it is not true that the set of all such quadrics forms a single congruence family, at least for $k \leq \frac{m}{2}-1$, as the following example shows:

Choose a $(k+2)$-dimensional subspace $W \subset V\left(A_{0}\right)$ and put $V:=W \oplus J W$. Then $A_{0} \mid V$ is a full conjugation on $V$, and therefore $Q^{\prime}:=Q\left(A_{0} \mid V, V\right)$ is a $k$-dimensional complex quadric contained in $Q$. It is easy to see that $Q^{\prime}$ is totally geodesic in $Q$. On the other hand, $Q$ contains a $(k+1)$-dimensional projective subspace $\Lambda$, as we will see in Section 3.2, and $\Lambda$ in turn contains a $k$-dimensional quadric $Q^{\prime \prime}$. However, $Q^{\prime \prime}$ is not totally geodesic in $Q$, because otherwise it would also be totally geodesic in $\Lambda$. Therefore, $Q^{\prime}$ and $Q^{\prime \prime}$ cannot be congruent in $Q$.

However, the set of $k$-dimensional complex quadrics which are totally geodesic submanifolds of $Q$ forms a congruence family:

\footnotetext{
${ }^{6}$ Suppose $M, N$ are Hermitian manifolds. Then we call a diffeomorphism $f: M \rightarrow N$ antiholomorphic if its differential is anti-linear.

${ }^{7}$ We exclude the case $m=2$ because the two-dimensional quadric $Q^{2}$ is holomorphically homothetic to $\mathbb{P}^{1} \times \mathbb{P}^{1}$, and therefore there are isometries on $Q^{2}$ which do not come from an isometry of $\mathbb{P}^{3}$.
} 
TheOREM 7. (a) A quadric $Q^{\prime} \in \mathfrak{F}\left(Q^{k}, \mathbb{P}(\mathbb{V})\right)$ is a totally geodesic submanifold of $Q$ if and only if there exists an $A \in \operatorname{Con}_{k+2}(\mathbb{V})$ such that $Q^{\prime}=Q(A, \mathbb{V})$ and $V(A) \subset V\left(A_{0}\right)$.

(b) The set of $k$-dimensional complex quadrics which are totally geodesic submanifolds of $Q$ forms a congruence family, which we denote by $\mathfrak{F}\left(Q_{t g}^{k}, Q\right)$. Already $\operatorname{SO}\left(V\left(A_{0}\right)\right)$ acts transitively on $\mathfrak{F}\left(Q_{t g}^{k}, Q\right)$ via the action $\left(B, Q^{\prime}\right) \mapsto \underline{B}\left(Q^{\prime}\right)$, and therefore we consider $\mathfrak{F}\left(Q_{t g}^{k}, Q\right)$ as a naturally reductive homogeneous $\mathrm{SO}\left(V\left(A_{0}\right)\right)$-space via Remark 2 . As such, $\mathfrak{F}\left(Q_{t g}^{k}, Q\right)$ is isomorphic to the real Grassmannian $G_{k+2}\left(V\left(A_{0}\right)\right)$. In particular, the reductive structure of $\mathfrak{F}\left(Q_{t g}^{k}, Q\right)$ is induced by a symmetric structure. We have $\operatorname{dim} \mathfrak{F}\left(Q_{t g}^{k}, Q\right)=(k+2)(m-k)$.

(c) $\mathfrak{F}\left(Q_{t g}^{k}, Q\right)$ is a compact, connected submanifold of $\mathfrak{F}\left(Q^{k}, \mathbb{P}(\mathbb{V})\right)$. As a naturally reductive homogeneous space, it is a subspace of $\mathfrak{F}\left(Q^{k}, \mathbb{P}(\mathbb{V})\right)$, and therefore, it is a totally geodesic submanifold of $\mathfrak{F}\left(Q^{k}, \mathbb{P}(\mathbb{V})\right)$.

Proof. (a) This fact is a consequence of the first author's study of totally geodesic submanifolds of $Q$; for the case $k=m-1$ also see [Re], Proposition 5. This study, which will be published in his dissertation, is based on the geometric description of the root spaces of the symmetric space $Q$ given in [Re], Theorem 3. Another discussion of totally geodesic submanifolds of $Q$ is found in $[\mathrm{CN}]$.

(b) Three spaces are of importance now, namely $\mathfrak{F}\left(Q_{t g}^{k}, Q\right), G_{k+2}\left(V\left(A_{0}\right)\right)$ and the set $\operatorname{Con}_{k+2}\left(\mathbb{V}, A_{0}\right):=\left\{A \in \operatorname{Con}_{k+2}(\mathbb{V}) \mid V(A) \subset V\left(A_{0}\right)\right\}$. The latter provides a connection between the first two spaces, namely by the maps

$$
\begin{aligned}
f_{1}: \operatorname{Con}_{k+2}\left(\mathbb{V}, A_{0}\right) & \rightarrow \mathfrak{F}\left(Q_{t g}^{k}, Q\right), A \mapsto Q(A, \mathbb{V}) \\
\text { and } \quad f_{2}: \operatorname{Con}_{k+2}\left(\mathbb{V}, A_{0}\right) & \rightarrow G_{k+2}\left(V\left(A_{0}\right)\right), A \mapsto V(A) .
\end{aligned}
$$

These maps turn out to be bijections. On the other hand, $G^{\prime}:=\operatorname{SO}\left(V\left(A_{0}\right)\right)$ acts on each of these spaces: on $\mathfrak{F}\left(Q_{t g}^{k}, Q\right)$ as described in (b), on $G_{k+2}\left(V\left(A_{0}\right)\right)$ as usual, and on $\operatorname{Con}_{k+2}\left(\mathbb{V}, A_{0}\right)$ by $(B, A) \mapsto B A B^{-1}$. With respect to these actions $f_{1}$ and $f_{2}$ are equivariant, therefore $f:=f_{1} \circ f_{2}^{-1}$ is an equivariant bijection from the Riemannian symmetric $G^{\prime}$-space $G_{k+2}\left(V\left(A_{0}\right)\right)$ onto the $G^{\prime}$-space $\mathfrak{F}\left(Q_{t g}^{k}, Q\right)$. In particular, $G^{\prime}$ acts transitively on $\mathfrak{F}\left(Q_{t g}^{k}, Q\right)$; moreover the symmetric space structure of $G_{k+2}\left(V\left(A_{0}\right)\right)$ can be pushed forward via $f$ onto $\mathfrak{F}\left(Q_{t g}^{k}, Q\right)$, which in particular gives the formula for the dimension.

By the same argument as in the proof of Theorem 6(b)(ii), we see that the symmetric space structure on $\mathfrak{F}\left(Q_{t g}^{k}, Q\right)$ induces the same naturally reductive structure as the one constructed via Section 1.3.

(c) Via complexification we consider $G^{\prime}$ as a subgroup of $G:=\mathrm{SU}(\mathbb{V})$. Then the inclusion $\mathfrak{F}\left(Q_{t g}^{k}, Q\right) \hookrightarrow \mathfrak{F}\left(Q^{k}, \mathbb{P}(\mathbb{V})\right)$ is equivariant and therefore $\mathfrak{F}\left(Q_{t g}^{k}, Q\right)$ can be considered as a homogeneous $G^{\prime}$-subspace of the homogeneous $G$-space $\mathfrak{F}\left(Q^{k}, \mathbb{P}(\mathbb{V})\right)$. As $G_{k+2}\left(V\left(A_{0}\right)\right)$ is compact, $\mathfrak{F}\left(Q_{t g}^{k}, Q\right)$ is a compact, regular submanifold of $\mathfrak{F}\left(Q^{k}, \mathbb{P}(\mathbb{V})\right)$.

Now, we fix $A \in \operatorname{Con}_{k+2}\left(\mathbb{V}, A_{0}\right)$. From the isomorphism $\mathfrak{F}\left(Q_{t g}^{k}, Q\right) \cong G_{k+2}\left(V\left(A_{0}\right)\right)$ we know the "symmetric space splitting" $\mathfrak{g}^{\prime}=\mathfrak{k}^{\prime} \oplus \mathfrak{m}^{\prime}$ of the Lie algebra $\mathfrak{g}^{\prime}=\mathfrak{o}\left(V\left(A_{0}\right)\right)$ with respect to $Q(A, \mathbb{V})$. In block matrix notation with respect to the decomposition $V\left(A_{0}\right)=V(A) \oplus \operatorname{ker} A$, we have 


$$
\mathfrak{m}^{\prime}=\left\{\left(\begin{array}{cc}
0 & -Z^{*} \\
Z & 0
\end{array}\right) \mid Z: V(A) \rightarrow \operatorname{ker} A \text { linear }\right\} .
$$

Thus we see $\mathfrak{m}^{\prime} \subset \mathfrak{m}_{1} \subset \mathfrak{m}$ (where $\mathfrak{m}$ is the reductive structure of $\mathfrak{F}\left(Q^{k}, \mathbb{P}(\mathbb{V})\right)$ and $\mathfrak{m}_{1}$ is as in the proof of Theorem 6(b)(i)). Moreover, the Killing forms of $\mathfrak{o}\left(V\left(A_{0}\right)\right)$ and of $\mathfrak{s u}(\mathbb{V})$ are given by $(X, Y) \mapsto-m \cdot\langle\langle X, Y\rangle\rangle \operatorname{resp} .(X, Y) \mapsto-2(m+2) \cdot\langle\langle X, Y\rangle\rangle$; here $\langle\langle\cdot, \cdot\rangle\rangle$ denotes the usual scalar product of endomorphisms (see [IT], p. 60). Hence $\mathfrak{F}\left(Q_{t g}^{k}, Q\right)$ is a naturally reductive homogeneous subspace of $\mathfrak{F}\left(Q^{k}, \mathbb{P}(\mathbb{V})\right)$; more precisely, the Riemannian metric of $\mathfrak{F}\left(Q_{t g}^{k}, Q\right)$ is the $\frac{m}{2(m+2)}$-fold of the Riemannian metric induced by $\mathfrak{F}\left(Q^{k}, \mathbb{P}(\mathbb{V})\right)$.

3.2. Projective subspaces in $Q$. We continue to use the notations of the previous section. To study the family of $k$-dimensional projective subspaces contained in $Q$ (such a subspace is automatically a totally geodesic submanifold of $Q$ ), we need to characterize the position of these spaces.

For this purpose, we introduce the quadratic cone

$$
C:=C\left(A_{0}, \mathbb{V}\right):=\left\{v \in \mathbb{V} \mid\left\langle v, A_{0} v\right\rangle_{\mathbb{C}}=0\right\}
$$

corresponding to $Q$ and call a complex linear subspace $W \subset \mathbb{V}$ isotropic, if $W \subset C$. We denote the set of $\ell$-dimensional complex isotropic subspaces of $\mathbb{V}$ by $G_{\ell}(\mathbb{V}, C)$. Note that $Q=G_{1}(\mathbb{V}, C)$.

For $W \in G_{\ell}(\mathbb{V}, C)$, we even have $\left\langle w_{1}, A_{0} w_{2}\right\rangle_{\mathbb{C}}=0$ for every $w_{1}, w_{2} \in W$. It can also be shown that there is a partial complex structure $j$ on $V\left(A_{0}\right)$ (i.e. $j$ is skew-adjoint with $\left.j^{3}=-j\right)$ of $\operatorname{rank} 2 \ell$ so that $W=\left\{x+J j x \mid x \in j\left(V\left(A_{0}\right)\right)\right\}$. Conversely, any space $\left\{x+J j x \mid x \in j\left(V\left(A_{0}\right)\right)\right\}$, where $j$ is some partial complex structure on $V\left(A_{0}\right)$, is isotropic. Thus, isotropic spaces may be interpreted geometrically as vector subspaces lying in a diagonal position with respect to the splitting $\mathbb{V}=V\left(A_{0}\right) \oplus J V\left(A_{0}\right)$. For such a space, we necessarily have $\operatorname{dim}_{\mathbb{C}} W \leq \frac{1}{2} \operatorname{dim}_{\mathbb{C}} \mathbb{V}$.

TheOrem 8. (a) For $\Lambda \in \mathfrak{F}\left(\mathbb{P}^{k}, \mathbb{P}(\mathbb{V})\right)$, we have $\Lambda \subset Q$ if and only if $\hat{\Lambda} \in$ $G_{k+1}(\mathbb{V}, C)$. This is possible only for $2 k \leq m$, and therefore we consider only this case in the sequel. $I(Q)$ acts transitively on the set of $k$-dimensional projective subspaces contained in $Q$, and therefore this set forms a congruence family, which we denote by $\mathfrak{F}\left(\mathbb{P}^{k}, Q\right){ }^{8}$ In fact, already $\mathrm{O}\left(V\left(A_{0}\right)\right)$ acts transitively on $\mathfrak{F}\left(\mathbb{P}^{k}, Q\right)$ via $(B, \Lambda) \mapsto \underline{B}(\Lambda)$.

(b) $\mathfrak{F}\left(\mathbb{P}^{k}, Q\right)$ is a complex, compact submanifold of $\mathfrak{F}\left(\mathbb{P}^{k}, \mathbb{P}(\mathbb{V})\right)$ of complex codimension $\frac{1}{2} \cdot(k+1)(k+2)$.

(c) If $2 k<m$, the manifold $\mathfrak{F}\left(\mathbb{P}^{k}, Q\right)$ is connected; if $2 k=m$, it consists of exactly two connected components. In either case, $\mathrm{SO}\left(V\left(A_{0}\right)\right)$ acts transitively on the connected components of $\mathfrak{F}\left(\mathbb{P}^{k}, Q\right)$, and they will therefore be considered as naturally reductive homogeneous $\mathrm{SO}\left(V\left(A_{0}\right)\right)$-spaces in the way described in Section 1.3, see Remark 2. Considered as homogeneous spaces only, they are subspaces of the homogeneous $\mathrm{SU}(\mathbb{V})$-space $\mathfrak{F}\left(\mathbb{P}^{k}, \mathbb{P}(\mathbb{V})\right.$ ) (but see (ii)). Furthermore:

${ }^{8} \mathfrak{F}\left(\mathbb{P}^{k}, Q\right)$ is isomorphic to the typical fibre of a twistor bundle, see [Ra], Proposition 2.1 (p. 88) and p. 102. 
(i) If $2 k=m$, the reductive structures of the connected components of $\mathfrak{F}\left(\mathbb{P}^{k}, Q\right)$ are induced by symmetric structures, and in this regard they are isomorphic to the irreducible Riemannian symmetric space $\mathrm{SO}(m+2) / \mathrm{U}(k+1)$ of type DIII, see [H], p. 518. Moreover, they are symmetric subspaces and therefore totally geodesic submanifolds of the symmetric space $\mathfrak{F}\left(\mathbb{P}^{k}, \mathbb{P}(\mathbb{V})\right)$.

(ii) If $1 \leq 2 k<m$, the reductive structure of $\mathfrak{F}\left(\mathbb{P}^{k}, Q\right)$ is not induced by a symmetric structure. Therefore, $\mathfrak{F}\left(\mathbb{P}^{k}, Q\right)$ (equipped with the reductive structure mentioned above) is also not a reductive homogeneous subspace of $\mathfrak{F}\left(\mathbb{P}^{k}, \mathbb{P}(\mathbb{V})\right)$.

Proof. (a) The given characterization of $k$-dimensional projective subspaces contained in $Q$ follows from the fact that $Q=G_{1}(\mathbb{V}, C)$. Using the representation of isotropic vector spaces by a partial complex structure and the description of $I(Q)$ given above, one sees that $I(Q)$ acts transitively on the set of $k$-dimensional projective subspaces contained in $Q$. Similarly, one sees that already $\mathrm{O}\left(V\left(A_{0}\right)\right)$ acts transitively on $\mathfrak{F}\left(\mathbb{P}^{k}, Q\right)$.

(b) Since $\mathrm{O}\left(V\left(A_{0}\right)\right)$ acts transitively on $\mathfrak{F}\left(\mathbb{P}^{k}, Q\right)$, this family is compact. We now show that it is a complex submanifold of $\mathfrak{F}\left(\mathbb{P}^{k}, \mathbb{P}(\mathbb{V})\right)$. Because of (a), the biholomorphic map $\theta^{-1}: \mathfrak{F}\left(\mathbb{P}^{k}, \mathbb{P}(\mathbb{V})\right) \rightarrow G_{k+1}(\mathbb{V})$ maps $\mathfrak{F}\left(\mathbb{P}^{k}, Q\right)$ onto $G_{k+1}(\mathbb{V}, C)$. Therefore, it is sufficient to show that $G_{k+1}(\mathbb{V}, C)$ is a complex submanifold of $G_{k+1}(\mathbb{V})$. We abbreviate $r:=k+1$.

Consider the Stiefel manifold $\mathrm{St}_{r}(\mathbb{V})$ of complex $r$-frames in $\mathbb{V}$ and the canonical projection $\sigma: \operatorname{St}_{r}(\mathbb{V}) \rightarrow G_{r}(\mathbb{V}),\left(a_{1}, \ldots, a_{r}\right) \mapsto \operatorname{span}\left\{a_{1}, \ldots, a_{r}\right\} . \operatorname{St}_{r}(\mathbb{V})$ is an open subset of $\mathbb{V}^{r}$ and therefore a complex manifold, and $\sigma$ is a holomorphic fibre bundle. If $\alpha(v, w):=$ $\left\langle v, A_{0} w\right\rangle_{\mathbb{C}}$ is the bilinear form defined by $A_{0}$, and

$$
g: \mathrm{St}_{r}(\mathbb{V}) \rightarrow M_{\text {symm }}(r \times r, \mathbb{C}),\left(a_{1}, \ldots, a_{r}\right) \mapsto\left(\alpha\left(a_{j}, a_{\ell}\right)\right)_{j, \ell=1, \ldots, r}
$$

(here, $M_{\text {symm }}(r \times r, \mathbb{C})$ is the space of symmetric $(r \times r)$-matrices over $\left.\mathbb{C}\right)$, we have $\sigma^{-1}\left(G_{r}(\mathbb{V}, C)\right)=g^{-1}(\{0\})$. Because $g$ is a holomorphic submersion, it follows that $\sigma^{-1}\left(G_{r}(\mathbb{V}, C)\right)$ is a complex submanifold of $\operatorname{St}_{r}(\mathbb{V})$ (see $[\mathrm{Na}]$, Corollary 2.5.5, p. 81). We now see by considering local sections of the holomorphic fibre bundle $\sigma$ that $G_{r}(\mathbb{V}, C)$ is a complex submanifold of $G_{r}(\mathbb{V})$; therefrom we also deduce the dimension formula.

(c) We consider an arbitrary subspace $\Lambda_{0} \in \mathfrak{F}\left(\mathbb{P}^{k}, Q\right)$, and denote by $j_{0}$ the partial complex structure on $V\left(A_{0}\right)$ of rank $2(k+1)$ such that $W_{0}:=\widehat{\Lambda_{0}}=\left\{x+J j_{0} x \mid x \in\right.$ $\left.j_{0}\left(V\left(A_{0}\right)\right)\right\}$.

Because $\mathrm{O}\left(V\left(A_{0}\right)\right)$ has exactly two connected components, $\mathfrak{F}\left(\mathbb{P}^{k}, Q\right)$ has at most two connected components, and $G:=\mathrm{SO}\left(V\left(A_{0}\right)\right)$ acts transitively on each of them, so they are homogeneous $G$-spaces, and become naturally reductive homogeneous $G$-spaces by the construction described in Proposition 4 and Remark 2. $\mathfrak{F}\left(\mathbb{P}^{k}, Q\right)$ is connected if and only if the isotropy group $K$ of the action of $\mathrm{O}\left(V\left(A_{0}\right)\right)$ on $\mathfrak{F}\left(\mathbb{P}^{k}, Q\right)$ at $\Lambda_{0}$, which can also be described as $K=\left\{B \in \mathrm{O}\left(V\left(A_{0}\right)\right) \mid B j_{0}=j_{0} B\right\}$, is not contained in the neutral component $G$ of $\mathrm{O}\left(V\left(A_{0}\right)\right)$. In the case $2 k<m$, if $B \in \mathrm{O}\left(V\left(A_{0}\right)\right)$ is the reflection in a 1codimensional subspace of $V\left(A_{0}\right)$ containing $j_{0}\left(V\left(A_{0}\right)\right)$, we have $B \in K$ and $\operatorname{det} B=-1$, so $K$ is not contained in $G$. On the other hand, in the case $2 k=m, K$ is the unitary group $\mathrm{U}\left(V\left(A_{0}\right), j_{0}\right)$ and hence connected, therefore $K$ is contained in $G$.

Via complexification, $G$ may be considered as a subgroup of $\mathrm{SU}(\mathbb{V})$, so the connected components of $\mathfrak{F}\left(\mathbb{P}^{k}, Q\right)$ are homogeneous subspaces of the $\mathrm{SU}(\mathbb{V})$-space $\mathfrak{F}\left(\mathbb{P}^{k}, \mathbb{P}(\mathbb{V})\right)$. 
(c)(i) By Theorem 5, the space $\mathfrak{F}\left(\mathbb{P}^{k}, \mathbb{P}(\mathbb{V})\right)$ is isomorphic to the complex Grassmannian $G_{k+1}(\mathbb{V})$. Therefore, $(\mathrm{SU}(\mathbb{V}), \widehat{K}, \widehat{\sigma})$ is a symmetric datum for $\mathfrak{F}\left(\mathbb{P}^{k}, \mathbb{P}(\mathbb{V})\right)$, where $\widehat{\sigma}$ is the involutive group automorphism $\mathrm{SU}(\mathbb{V}) \rightarrow \mathrm{SU}(\mathbb{V}), B \mapsto S B S^{-1}$ defined by using the reflection $S: \mathbb{V} \rightarrow \mathbb{V}$ in $W_{0}$, and $\widehat{K}=\mathrm{S}\left(\mathrm{U}\left(W_{0}\right) \times \mathrm{U}\left(W_{0}^{\perp}\right)\right)$ is exactly the group of fixed points of $\widehat{\sigma}$.

One can show that for any $B \in G$, we have $\widehat{\sigma}(B)=j_{0}^{-1} B j_{0} \in G$ (here and in the sequel, we once again interpret the elements of $G$ as elements of $\mathrm{SU}(\mathbb{V})$ via complexification), so $\sigma:=\widehat{\sigma} \mid G$ is an involutive automorphism of $G$. The above description of $\widehat{\sigma} \mid G$ also implies that for any $B \in G$ we have: $B \in \widehat{K}=\operatorname{Fix}(\widehat{\sigma})$ if and only if $j_{0} B=B j_{0}$; and therefore $\widehat{K} \cap G=K$. Thus, $(G, K, \sigma)$ is a symmetric datum for the connected component of $\mathfrak{F}\left(\mathbb{P}^{k}, Q\right)$ containing $\Lambda_{0}$, which thereby becomes a symmetric subspace of the symmetric space $\mathfrak{F}\left(\mathbb{P}^{k}, \mathbb{P}(\mathbb{V})\right)$.

It remains to show that the reductive structure $\mathfrak{m}_{\sigma}:=\left\{X \in \mathfrak{g} \mid \sigma_{L}(X)=-X\right\}$ thus induced on the connected component of $\mathfrak{F}\left(\mathbb{P}^{k}, Q\right)$ is in fact equal to the naturally reductive structure $\mathfrak{m}$ from Section 1.3, and this follows by the same argument as in the proof of Theorem 6(b)(ii).

(c)(ii) Once again, we abbreviate $r:=k+1$; then we have $4 \leq 2 r<m+2$.

First, we determine the reductive structure $\mathfrak{m}$ of $\mathfrak{F}\left(\mathbb{P}^{k}, Q\right)$ at $\Lambda_{0} \in \mathfrak{F}\left(\mathbb{P}^{k}, Q\right)$ : Now, the isotropy group of $G$ at $\Lambda_{0}$ is $K \cap G=\left\{B \in G \mid B j_{0}=j_{0} B\right\}$; hence its Lie algebra is $\mathfrak{k}:=\left\{X \in \mathfrak{g} \mid X j_{0}=j_{0} X\right\}=\operatorname{ker} \operatorname{ad}\left(j_{0}\right)$, note that $j_{0} \in \mathfrak{g}$. Because $\operatorname{ad}\left(j_{0}\right)$ is skew-adjoint with respect to the Killing form of $\mathfrak{g}$, therefore the reductive structure of $\mathfrak{F}\left(\mathbb{P}^{k}, Q\right)$ is given by $\mathfrak{m}=\operatorname{ad}\left(j_{0}\right)(\mathfrak{g})$.

We now apply the foregoing results to a specific choice of $\Lambda_{0}$ resp. $j_{0}$ : Fixing an orthonormal basis $\left(a_{1}, \ldots, a_{m+2}\right)$ of $V\left(A_{0}\right)$, we define a partial complex structure $j_{0}$ of rank $2 r$ by

$$
\forall \nu \in\{1, \ldots, m+2\}: j_{0} a_{\nu}= \begin{cases}a_{\nu+r} & \text { for } 1 \leq \nu \leq r \\ -a_{\nu-r} & \text { for } r+1 \leq \nu \leq 2 r \\ 0 & \text { for } 2 r+1 \leq \nu \leq m+2\end{cases}
$$

and consider the corresponding element $\Lambda_{0}=\left[W_{0}\right] \in \mathfrak{F}\left(\mathbb{P}^{k}, Q\right)$. Further, we define endomorphisms $X, Y \in \mathfrak{g}$ by

$$
\begin{aligned}
& X a_{1}=a_{r}, X a_{r}=-a_{1}, X a_{\nu}=0 \text { otherwise } \\
& \text { and } Y a_{2 r}=-a_{2 r+1}, Y a_{2 r+1}=a_{2 r}, Y a_{\nu}=0 \text { otherwise. }
\end{aligned}
$$

Then we have $X^{\prime}:=\operatorname{ad}\left(j_{0}\right) X, Y^{\prime}:=\operatorname{ad}\left(j_{0}\right) Y \in \mathfrak{m}$. We further put $Z:=\left[X^{\prime}, Y^{\prime}\right]$. Then, a simple calculation shows $\left(\operatorname{ad}\left(j_{0}\right) Z\right) a_{1}=a_{2 r+1}$, and therefore $Z \notin \operatorname{ker} \operatorname{ad}\left(j_{0}\right)=\mathfrak{k}$. Thus we have $[\mathfrak{m}, \mathfrak{m}] \not \subset \mathfrak{k}$, and therefore, the reductive structure of $\mathfrak{F}\left(\mathbb{P}^{k}, Q\right)$ cannot come from a symmetric structure. Furthermore, because $\mathfrak{F}\left(\mathbb{P}^{k}, \mathbb{P}(\mathbb{V})\right)$ is a symmetric space, the submanifold $\mathfrak{F}\left(\mathbb{P}^{k}, Q\right)$ cannot be a reductive homogeneous subspace.

\section{References}

[B] N. Bourbaki, Variétés différentielles et analytiques : Fascicule de résultats, Hermann, Paris, 1967. 
[CN] B.-Y. Chen and T. Nagano, Totally geodesic submanifolds of symmetric spaces, I, Duke Math. J. 44 (1977), 745-755.

[GH] P. Griffiths and J. Harris, Principles of Algebraic Geometry, Wiley, New York, 1978.

$[\mathrm{H}] \quad$ S. Helgason, Differential Geometry, Lie Groups, and Symmetric Spaces, Academic Press, New York, 1978.

[IT] M. Ise and M. Takeuchi, Theory of Lie Groups, American Mathematical Society, Providence, Rhode Island, 1991.

[K] S. Kobayashi, Transformation Groups in Differential Geometry, Springer, Berlin, 1972.

$[\mathrm{KN}] \quad$ S. Kobayashi and K. Nomizu, Foundations of Differential Geometry, volume II, Interscience Publications, New York, 1969.

[NT] H. Naitoh and M. Takeuchi, Symmetric submanifolds of symmetric spaces, Sugaku Exp. 2 (1989), 157-188.

[Na] R. Narasimhan, Analysis on Real and Complex Manifolds, Masson, Paris, 1968.

[No] K. Nomizu, Invariant affine connections on homogeneous spaces, Amer. J. Math. 76 (1954), 33-65.

[Ra] J. Rawnsley, $f$-structures, $f$-twistor spaces and harmonic maps, Lecture Notes in Mathematics (Geometry Seminar "Luigi Bianchi" II), 1164 (1984).

[Re] H. Reckziegel, On the geometry of the complex quadric, in Geometry and Topology of Submanifolds VIII, Dillen et al. (eds.), World Scientific, 1996, 302-315.

[V] V. S. Varadarajan, Lie Groups, Lie Algebras and Their Representations, Englewood Cliffs, New Jersey, 1974. 